\title{
A RANDOM TROTTER PRODUCT FORMULA
}

\author{
THOMAS G. KURTZ
}

Abstract. Let $X(t)$ be a pure jump process with state space $S$ and let $\xi_{0}, \xi_{1}, \xi_{2}, \cdots$ be the succession of states visited by $X(t)$, $\Delta_{0} \Delta_{1} \cdots$ the sojourn times in each state, $N(t)$ the number of transitions before $t$ and $\Delta_{t}=t-\sum_{k=0}^{N(t)-1} \Delta_{k}$. For each $x \in S$ let $T_{x}(t)$ be an operator semigroup on a Banach space $L$. Define $T_{\lambda}(t, w)=$ $T_{\xi_{0}}\left((1 / \lambda) \Delta_{0}\right) T_{\xi_{1}}\left((1 / \lambda) \Delta_{1}\right) \cdots T_{\xi_{N(\lambda t)}}\left((1 / \lambda) \Delta_{\lambda t}\right)$. Conditions are given under which $T_{\lambda}^{1}(t, w)$ will converge almost surely (or in probability) to a semigroup of operators as $\lambda \rightarrow \infty$. With $S=\{1,2\}$ and

$$
\begin{aligned}
X(t) & =1, & & 2 n \leqq t<2 n+1, \\
& =2, & & 2 n+1 \leqq t<2 n+2,
\end{aligned}
$$

$n=0,1,2, \cdots$ the result is just the "Trotter product formula".

1. Introduction. Let $X(t)$ be a stochastic process with values in a separable, locally compact metric state space $S$. Of course $X(t)$ is a function from a sample space $\Omega$ into $S$. We will assume that $\Omega=D_{S}(0, \infty)$, the space of right continuous functions with left hand limits taking values in $S$ and $X(t, w)=w(t)$.

Furthermore we will assume that $X(t)$ is a pure jump process; that is, there is a set $N \subset \Omega$ with $P(N)=0$ such that for every pair $(t, w), w \notin N$, $X(t, w)=X(t+s, w)$ for all sufficiently small $s>0$, and $X(t, w)$ has only a finite number of discontinuities in a finite time interval. Under this assumption it makes sense to talk about $\xi_{0}, \xi_{1}, \xi_{2}, \cdots$, the sequence of states visited, and $\Delta_{0} \Delta_{1} \cdots$, the sojourn times in these states. In addition we define $N(t)$ to be the number of transitions before time $t$ and

$$
\Delta_{t}=t-\sum_{k=0}^{N(t)-1} \Delta_{k} .
$$

For each $x \in S$ let $T_{x}(t)$ be a semigroup of linear operators on a Banach space $L$ with infinitesimal operator $A_{x}$, satisfying $\left\|T_{x}(t)\right\| \leqq e^{\alpha t}$ for some

Received by the editors November 22, 1971.

AMS 1970 subject classifications. Primary 47D05; Secondary 60J99, 34F05, 34G05.

Key words and phrases. Operator semigroup, random evolution, Markov process, Trotter product. 
fixed $\alpha$. We define the random evolution governed by $X(\lambda t)$ by

$$
T_{\lambda}(t, w)=T_{\xi_{0}}\left(\frac{1}{\lambda} \Delta_{0}\right) T_{\xi_{1}}\left(\frac{1}{\lambda} \Delta_{1}\right) \cdots T_{\xi_{N(\lambda t)}}\left(\frac{1}{\lambda} \Delta_{\lambda t}\right) .
$$

The definition of a random evolution, originally given by Griego and Hersh [1] for $X(t)$ a Markov chain, can perhaps best be motivated in the following way:

For each $x \in S$, let $P_{x}(t, y, \Gamma)$ be a Markov transition function on a measurable state space $(E, \mathscr{B})$, and let $T_{x}(t)$ be the corresponding semigroup on $B(E, \mathscr{B})$, the space of bounded measurable functions. Let $Z$ be the process (assuming one exists) whose development is governed by $\boldsymbol{P}_{x}(t, y, \Gamma)$ on time intervals in which $X$ is in state $x$. Then, at least intuitively,

$$
E(f(Z(t)) \mid X(s), s \leqq t, Z(0)=z)=\left(T_{\xi_{0}}\left(\Delta_{0}\right) T_{\xi_{1}}\left(\Delta_{1}\right) \cdots T_{\xi_{N}(t)}\left(\Delta_{t}\right) f\right)(z)
$$

We are interested in the behavior of $T_{\lambda}(t, w)$ as $\lambda$ tends to infinity, that is, in what happens if the mode of development of the random evolution (or the process $Z$ ) changes at a very rapid rate.

In $\S 2$, we will give conditions under which $T_{\lambda}(t, w)$ converges almost surely to a semigroup whose infinitesimal operator is the closure of $\int A_{x} f \mu(d x)$ where $\mu$ satisfies

$$
\lim _{t \rightarrow \infty} \frac{1}{t} \int_{0}^{t} g(X(s)) d s=\int g(x) \mu(d x)
$$

for all bounded continuous $g$.

Griego and Hersh [1] and Hersh and Pinsky [2] consider the case where $\int A_{x} f \mu(d x)=0$ (i.e. the limiting semigroup is the identity) and $X(t)$ is a finite Markov chain. They give limit theorems for $E\left(T_{\lambda}(\lambda t, w)\right)$ under the assumption that the $T_{x}(t)$ commute. In a subsequent paper we will show that many of their results hold without the assumption of commutativity.

In what follows we will use a number of different Banach spaces. We will use subscripts on the norm notation only when there is a possibility of confusion (e.g. the norm on $L$ will be denoted by $\|\cdot\|_{L}$ ).

\section{The limit theorem.}

THEOREM (2.1). Let $X(t)$ be a pure jump process with state space $S$. Suppose $S$ is a separable, locally compact metric space and there is a measure $\mu$ on the Borel subsets of $S$ such that $\mu(S)=1$ and

$$
P\left\{\lim _{t \rightarrow r} \frac{1}{t} \int_{0}^{t} g(X(s)) d s=\int g(x) \mu(d x)\right\}=1
$$

for every real, bounded, continuous function $g$. 
For each $x \in S$ let $T_{x}(t)$ be a semigroup of linear operators on a Banach space $L$ with infinitesimal operator $A_{x}$ satisfying $\left\|T_{x}(t)\right\| \leqq e^{x t}$, for some $\alpha$ independent of $x$.

Let $D$ be the set of $f \in L$ such that $A_{x} f: S \rightarrow L$ is a bounded continuous function of $x$. Define $A f=\int A_{x} f \mu(d x)$ for $f \in D$.

If $D$ is dense in $L$ and $\mathscr{R}(\mu-A)$ is dense in $L$ for some $\mu>\alpha$, then the closure of $A$ is the infinitesimal operator for a strongly continuous semigroup $T(t)$ defined on $L$ and

$$
P\left\{\lim _{\lambda \rightarrow \infty} T_{i}(t, w) f=T(t) f\right\}=1
$$

for every $f \in L$.

To prove Theorem (2.1) we will use the following which is a consequence of the results in [3].

Theorem (2.4). For $0<\lambda<\infty$, let $M_{\lambda}$ be a Banach space and $\mathscr{M}$ the Banach space of bounded functions $\lambda \rightarrow f(\lambda) \in M_{\lambda}$ with $\|f(\cdot)\|=\sup _{\lambda}\|f(\lambda)\|$. Let $\mathrm{LIM}_{\lambda \rightarrow \infty}$ denote any notion of limit (e.g. strong convergence, weak convergence) such that $P f(\cdot) \equiv \operatorname{LIM}_{\lambda \rightarrow \infty} f(\lambda)$ defines a bounded linear operator from the subspace of convergent functions into another Banach space $M$.

For each $\lambda$ let $S_{\lambda}(t)$ be a semigroup of linear operators on $M_{\lambda}$ with infinitesimal operator $B_{\lambda}$ satisfying $\left\|S_{\lambda}(t)\right\|<e^{x t}$ for some $\alpha$ independent of $\lambda$.

Suppose $\operatorname{LIM}_{\lambda \rightarrow \infty} f(\lambda)=0$ implies

$$
\operatorname{LIM}_{\lambda \rightarrow \infty} S_{\lambda}(t) f(\lambda)=0, \quad \text { all } t,
$$

and

$$
\underset{\lambda \rightarrow \infty}{\operatorname{LIM}}\left(\mu-B_{\lambda}\right)^{-1} f(\lambda) \equiv \underset{\lambda \rightarrow \infty}{\operatorname{LIM}} \int_{0}^{\infty} e^{-\mu t} S_{\lambda}(t) f(\lambda) d t=0 . \quad \text { all } \mu>\alpha .
$$

Let

$\mathscr{D}(A)=\{g \in M: \exists f(\cdot) \in \mathscr{M} \ni \underset{\lambda \rightarrow \infty}{\operatorname{LIM}} f(\lambda)=g$ and

( $A$ may be multivalued.)

$$
\left.\operatorname{LIM}_{\lambda \rightarrow \infty} B_{\lambda} f(\hat{\lambda}) \equiv A g \text { exists }\right\}
$$

If $\mathscr{D}(A)$ is dense in $M$ and $\mathscr{R}(\mu-A)$ is dense in $M$ for some $\mu>\alpha$, then the closure of $A$ is the infinitesimal operator of a strongly continuous semigroup $T(t)$ on $M$ and $\operatorname{LIM}_{\lambda \cdots \infty} f(\lambda)=g \in M$ implies $\operatorname{LIM}_{\lambda \rightarrow \infty} S_{\lambda}(t) f(\lambda)=$ $T(t) g$.

In our application of Theorem (2.4), $M_{\lambda}$ will be the space of bounded continuous functions from $D_{S}(0, \infty)$ into $L$ with $\|g\|=\sup _{u \in D_{S}}\|g(w)\|$ for all $\lambda>0$, and $M$ will be $L$. Let $\theta_{t}$ be the shift operator on $D_{S}(0, \infty)$, 
that is $\theta_{t} w(s)=w(s+t)$. We will say $\operatorname{LIM}_{\lambda \rightarrow \infty} f(\lambda, w)=g \in L \equiv M$ if

$$
P\left\{\lim _{i \rightarrow \infty} \sup _{s \leqq t}\left\|f\left(\lambda, \theta_{\lambda s} w\right)-g\right\|_{L}=0\right\}=1
$$

for all $t>0$. This notion of convergence is stronger than almost sure convergence and weaker than convergence uniform in $w$. Although we are only interested in almost sure convergence we need the extra strength in order to insure that (2.5) and (2.6) hold.

Finally, the semigroups $S_{\lambda}(t)$ are given by

$$
S_{\lambda}(t) f(\lambda, w) \equiv T_{\lambda}(t, w) f\left(\lambda, \theta_{\lambda t} w\right) .
$$

If $f(\lambda, w) \equiv f \in L$ we will write $S_{\lambda}(t) f$.

To complete the proof of Theorem (2.1) we prove the following series of lemmas, all under the assumptions of the theorem.

Lemma (2.9). For $f \in D$,

$$
\left\|T_{\lambda}(t, w) f-f\right\|_{L} \leqq t e^{\alpha t} \sup _{x}\left\|A_{x} f\right\|_{L}
$$

and hence, since $D$ is dense in $L$,

$$
\lim _{t \rightarrow 0} \sup _{w, \lambda}\left\|T_{\lambda}(t, w) g-g\right\|=0
$$

for all $g \in L$.

ProOF.

$$
\left\|T_{\lambda}(t, w) f-f\right\|_{L}
$$

$$
\begin{aligned}
\leqq & \sum_{k=0}^{N(\lambda t)-1}\left\|T_{\xi_{0}}\left(\frac{1}{\lambda} \Delta_{0}\right) \cdots T_{\xi k-1}\left(\frac{1}{\lambda} \Delta_{k-1}\right)\left(T_{\xi k}\left(\frac{1}{\lambda} \Delta_{k}\right)-I\right) f\right\|_{L} \\
& +\left\|T_{\xi 0}\left(\frac{1}{\lambda} \Delta_{0}\right) \cdots T_{\xi N(\lambda t)-1}\left(\frac{1}{\lambda} \Delta_{N(\lambda t)-1}\right)\left(T_{\xi N(\lambda t)}\left(\frac{1}{\lambda} \Delta_{\lambda t}\right)-I\right) f\right\|_{L} \\
\leqq & e^{\alpha t}\left(\sum_{k=0}^{N(\lambda t)-1} \frac{1}{\lambda} \Delta_{k}\left\|A_{\xi k} f\right\|_{L}+\frac{1}{\lambda} \Delta_{\lambda t}\left\|A_{\xi N(\lambda t)} f\right\|_{L}\right) \\
\leqq & t e^{\alpha t} \sup _{x}\left\|A_{x} f\right\|_{L} .
\end{aligned}
$$

Lemma (2.13). There is a function $\varepsilon(\lambda)$ satisfying $\lim _{\lambda \rightarrow \infty} \varepsilon(\lambda)=0$ and

$$
P\left\{\lim _{\lambda \rightarrow \infty} \sup _{t \geqq T}\left|\frac{1}{\varepsilon(\lambda)} \int_{t}^{t+\varepsilon(\lambda)} g(X(\lambda s, w)) d s-\int g(x) \mu(d x)\right|=0\right\}=1
$$

for every real, bounded continuous function $g$ and every $T>0$.

Proof. Since the claim is that certain linear functionals of norm one on the space of bounded continuous functions converge to a bounded linear 
functional of norm one that is given by a measure, it will suffice to prove the result for continuous functions vanishing at infinity. Since the space of continuous functions on $S$ vanishing at infinity is separable, we need only consider a countable dense subset, say $g_{1} g_{2} g_{3} \cdots$.

Note that

$$
\frac{1}{\varepsilon} \int_{t}^{t+\varepsilon} g(X(\lambda s, w)) d s=\frac{1}{\lambda \varepsilon} \int_{\lambda t}^{\lambda(t+\varepsilon)} g(X(s, w)) d s
$$

is uniformly continuous in $t$.

Consequently (2.2) implies

$$
P\left\{\lim _{\lambda \rightarrow \infty} \sup _{t \leqq T}\left|\frac{1}{\varepsilon} \int_{t}^{t+\varepsilon} g(X(\lambda s, w)) d s-\int g(x) \mu(d x)\right|=0\right\}=1,
$$

for every $g, \varepsilon>0$ and $T>0$. Let $\varepsilon_{n} \rightarrow 0, T_{n} \rightarrow \infty$ and $\delta_{n} \rightarrow 0$. Then there exists a $\lambda_{n}$ such that

$$
\sup _{i \leqq n} P\left\{\sup _{\lambda \geqq \lambda_{n}} \sup _{t \leqq T_{n}}\left|\frac{1}{\varepsilon_{n}} \int_{t}^{t+\varepsilon_{n}} g_{i}(X(\lambda s, w)) d s-\int g(x) \mu(d x)\right|>\delta_{n}\right\} \leqq \delta_{n} .
$$

The lemma follows by setting $\varepsilon(\lambda)=\varepsilon_{n}$ for $\lambda_{n} \leqq \lambda<\lambda_{n+1}$.

Lemma (2.16). Let $f \in D$. Then $g(w)=(1 / \varepsilon) \int_{0}^{\varepsilon} T_{\lambda}(s, w) f d s$ is in $\mathscr{D}\left(B_{\lambda}\right)$ and

$$
B_{\lambda} g(w)=\frac{1}{\varepsilon} \int_{0}^{\varepsilon} T_{\lambda}(s, w) A_{X(\lambda s, w)} f d s .
$$

Proof. The fact that $g(w) \in \mathscr{D}\left(B_{\lambda}\right)$ is a standard result of semigroup theory. The form of $B_{\lambda} g(w)$ is obtained from the following inequality.

$$
\begin{aligned}
\| \frac{1}{\varepsilon} \int_{0}^{\varepsilon} T_{\lambda}(s, w) & A_{X(\lambda s, w)} f d s-\frac{S_{\lambda}(t) g(w)-g(w)}{t} \|_{L} \\
& =\left\|\frac{1}{\varepsilon} \int_{0}^{\varepsilon} T_{\lambda}(s, w)\left(A_{X(\lambda s, w)} f-\frac{T_{\lambda}\left(t, \theta_{\lambda s} w\right) f-f}{t}\right) d s\right\|_{L} \\
& \leqq \frac{1}{\varepsilon} \int_{0}^{\varepsilon} e^{\alpha s}\left\|\left(A_{X(\lambda s, w)} f-\frac{T_{\lambda}\left(t, \theta_{\lambda s} w\right) f-f}{t}\right)\right\|_{L} d s
\end{aligned}
$$

Noting that $X(\lambda s, w)=X\left(0, \theta_{\lambda s} w\right)$, we observe

$$
\left\|A_{X(\lambda s, w)} f-\frac{T_{\lambda}\left(t, \theta_{\lambda, s} w\right) f-f}{t}\right\|_{L}
$$

is bounded by (2.10) and goes to zero as $t$ goes to zero for all $s$. The lemma then follows by the dominated convergence theorem. 
Lemma (2.19). Let $f \in D$. Define

Then

$$
f(\lambda, w)=\frac{1}{\varepsilon(\lambda)} \int_{0}^{\varepsilon(\lambda)} T_{\lambda}(s, w) f d s .
$$

and

$$
\underset{\lambda \rightarrow \infty}{\operatorname{LIM}} f(\lambda, w)=f
$$

$$
\operatorname{LIM}_{\lambda \rightarrow \infty} B_{\lambda}(\lambda, w)=\int A_{x} f \mu(d x) .
$$

Proof. We must show that

almost surely.

$$
\lim _{\lambda \rightarrow \infty} \sup _{t \leqq T}\left\|B_{\lambda} f\left(\lambda, \theta_{\lambda t} w\right)-\int A_{x} f \mu(d x)\right\|_{L}=0
$$

$$
\begin{aligned}
\sup _{t \leqq T}\left\|B_{\lambda} f\left(\lambda, \theta_{\lambda t} w\right)-\int A_{x} f \mu(d x)\right\|_{L} \\
=\sup _{t \leqq T}\left\|\frac{1}{\varepsilon(\lambda)} \int_{0}^{\varepsilon(\lambda)} T_{\lambda}\left(s, \theta_{\lambda t} w\right) A_{X\left(\lambda s, \theta_{\lambda t} w\right)} f-\int A_{x} f \mu(d x)\right\|_{L} \\
\leqq \sup _{t \leqq T}\left\|\frac{1}{\varepsilon(\lambda)} \int_{0}^{\varepsilon(\lambda)}\left(T_{\lambda}\left(s, \theta_{\lambda t} w\right)-I\right) A_{X\left(\lambda s, \theta_{\lambda t} w\right)} f d s\right\| \\
\quad+\sup _{t \leqq T}\left\|\frac{1}{\varepsilon(\lambda)} \int_{0}^{\varepsilon(\lambda)} A_{X\left(\lambda s, \theta_{\lambda t} w\right)} f d s-\int A_{x} f \mu(d x)\right\| .
\end{aligned}
$$

The second term on the right can be rewritten as

$$
\sup _{t \leqq T}\left\|\frac{1}{\varepsilon(\lambda)} \int_{t}^{t+\varepsilon(\lambda)} A_{X(\lambda s, w)} f d s-\int A_{x} f \mu(d x)\right\|
$$

and goes to zero almost surely by (2.14), the boundedness and continuity of $A_{x} f$ as a function of $x$, and the separability and local compactness of $S$.

The first term on the right of (2.20) can be bounded by

$$
\begin{aligned}
\sup _{t \leqq T} \sup _{x \in K}\left\|\frac{1}{\varepsilon(\lambda)} \int_{0}^{\varepsilon(\lambda)}\left(T_{\lambda}\left(s, \theta_{\lambda t} w\right)-I\right) A_{x} f\right\| \\
+\left(2 \sup \left\|A_{x} f\right\|\right)\left(\sup _{t \leqq T} \frac{1}{\varepsilon(\lambda)} \int_{0}^{\varepsilon(\lambda)} \chi_{K^{c}}\left(X\left(\lambda s, \theta_{\lambda t} w\right)\right) d s\right) .
\end{aligned}
$$

A compact set $K$ can be selected so that the lim sup of the second term on the right of (2.21) can be made arbitrarily small. Given a compact $K$ the first goes to zero by the continuity of $A_{x} f$, the compactness of $K$ and (2.11). 
Proof of Theorem (2.1). Lemma (2.19) implies that the operator $A$ in Theorem (2.4) is an extension of $A f=\int A_{x} f \mu(d x)$. Consequently, under the hypotheses of Theorem (2.1), Theorem (2.4) implies, for all $f \in L$ and $f(w) \equiv f$,

This implies (2.3).

$$
\operatorname{LIM}_{\lambda \rightarrow \infty} S_{\lambda}(t) f(w)=T(t) f
$$

REMARK. Since the probability measure in (2.2) is arbitrary, we have in fact proved convergence for every $w \in D_{S}(0, \infty)$ that is constant except for a discrete set of jumps and satisfies

$$
\lim _{t \rightarrow \infty} \frac{1}{t} \int_{0}^{t} g(w(t)) d t=\int g(x) \mu(d x)
$$

for all continuous $g$. Consequently Theorem (2.1) gives a generalization of the "Trotter product formula", that is

TheOREM (2.22) (TrotTer [4]). Suppose $T(t)$ and $S(t)$ are semigroups of linear operators on $L$, with infinitesimal operators $A$ and $B$, satisfying $\|T(t)\| \leqq e^{\alpha t}$ and $\|S(t)\| \leqq e^{\alpha t}$. If $\mathscr{D}(A) \cap \mathscr{W}(B)$ is dense in L and $\mathscr{R}\left(\mu-\frac{1}{2}(A+B)\right)$ is dense in $L$ for some $\mu>\alpha$ then the closure of $\frac{1}{2}(A+B)$ is the infinitesimal operator of a semigroup $U(t)$ on $L$ and

for all $f$ in $L$.

$$
\lim _{h \rightarrow 0}(T(h / 2) S(h / 2))^{[t / h]} f=U(t) f
$$

We observe that almost sure convergence in (2.2) and (2.3) can be replaced by convergence in probability with only minor alteration in the proof. In particular, the notion of convergence becomes: $\operatorname{LIM}_{\lambda \rightarrow \infty} f(\lambda, w)=$ $g \in L$ if

$$
\lim _{\lambda \rightarrow \infty} P\left\{\sup _{s \leq t}\left\|f\left(\lambda, \theta_{\lambda s} w\right)-g\right\|>\varepsilon\right\}=0
$$

for every $\varepsilon>0$ and every $t>0$.

EXAMPLE. Let $X(t)$ satisfy the conditions of Theorem (2.1). Let

$$
F(x, z): S \times R^{n} \rightarrow R^{n}
$$

be bounded and satisfy

for all $x_{0} \in S$, and

$$
\limsup _{x \rightarrow s_{0}}\left|F(x, z)-F\left(x_{0}, z\right)\right|=0
$$

$$
\sup _{x}\left|F\left(x, z_{1}\right)-F\left(x, z_{2}\right)\right|<M\left|z_{1}-z_{2}\right|
$$


for all $z_{1}, z_{2} \in R^{n}$ and some fixed $M$. Let $Z_{\lambda}(t, z)$ be the solution of

$$
Z_{\lambda}(t, z)=z+\int_{0}^{t} F\left(X(\lambda s), Z_{\lambda}(s, z)\right) d s .
$$

Theorem (2.1) implies

$$
P\left\{\limsup _{\lambda \rightarrow \infty}\left|Z_{\lambda}(t, z)-Z(t, z)\right|=0\right\}=1
$$

where $Z(t, z)$ is the solution of

$$
Z(t, z)=z+\int_{0}^{t} \int_{S} F(x, Z(s, z)) \mu(d x) d s .
$$

\section{REFERENCES}

1. R. Hersh and R. J. Griego, Random evolutions, Markov chains, and systems of partial differential equations, Proc. Nat. Acad. Sci. U.S.A. 62 (1969), 305-308. MR 42 \#5099.

2. R. Hersh and M. Pinsky, Random evolutions are asymptotically Gaussian, Comm. Pure. Appl. Math. 25 (1972), 33-44.

3. Thomas G. Kurtz, A general theorem on the convergence of operator semigroups, Trans. Amer. Math. Soc. 148 (1970), 23-32. MR 41 \#867.

4. H. F. Trotter, On the product of semi-groups of operators, Proc. Amer. Math. Soc. 10 (1959), 545-551. MR 21 \#7446.

Department of Mathematics, University of Wisconsin, Madison, Wisconsin 53706 